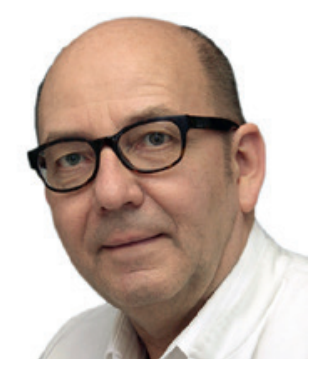

\title{
Uveitis in entzündlich-rheumatischen Erkrankungen
}

\author{
Uwe Pleyer \\ Klinik für Augenheilkunde, Charité - Universitätsmedizin Berlin, Campus Virchow Klinikum, Berlin, Deutschland
}

Abstract aus Hysa E, Cutolo CA, Gotelli E, Pacini G, Schenone C, Kreps EO, Smith V, Cutolo M. Immunopathophysiology and clinical impact of uveitis in inflammatory rheumatic diseases: An update. Eur J Clin Invest. 2021 Aug;51(8):e13572.

\section{Keywords}

Autoimmune rheumatic diseases · Behçet's disease · Connective tissue diseases · Sarcoidosis · Spondyloarthritis · Uveitis

\begin{abstract}
Background: Uveitis is one of the most frequent ophthalmologic manifestations in rheumatology. Uveal inflammation can underlie a systemic inflammatory rheumatic disease (SIRD) in approximately $30 \%$ of cases with a significant burden on the quality of life since it represents a cause of blindness in up to $20 \%$ of cases in Western countries.
\end{abstract}

Methods: In this review, we provide a comprehensive overview of the pathophysiology of uveitis associated with SIRDs. According to our literature survey on the epidemiology of uveitis among SIRDs, spondyloarthritides, Behçet's disease and sarcoidosis get the major impact.

Results: In Behçet's uveitis, the key players are highly polarized Th1 and Th17 lymphocytes, natural killer T cells and $\gamma \delta T$ cells. All contribute to a great destructive inflammatory environment with the most serious visual damage resulting from the involvement of the posterior segment of the eye. In contrast, spondyloarthritides-related uveitis derives from a complex interaction between genetic background and extra-ocular inflammatory mediators originating from enthesitis, arthritis, psoriatic lesions and microbiome pro-inflammatory alterations. In such conditions, the immune infiltration of CD4+ T cells, Th17 and natural killer cells along with pro-inflammatory cytokines, TNF-a among all, leads to intraocular inflammation. Lastly, granuloma formation represents the primary hallmark lesion in sarcoid uveitis. This suggests a profound link between the innate system that mainly recruits activated macrophages and adaptive system involving by Th1, Th17 and Th17.1 cells.

Conclusions: Awareness among rheumatologists of a potential severe ocular involvement generates new insights into targeted therapeutic approaches and personalized treatments for each patient. 


\section{Transfer in die Praxis}

\section{Hintergrund}

Inzwischen ist sie gut bekannt: die Verbindung zwischen intraokularer Entzündung und «Rheuma». Daher wird in der Regel der-Uveitis Patient mit der Frage konfrontiert: «Haben Sie Rheuma...?» Rheuma gilt als Volkskrankheit. Doch ist Rheuma keine eigenständige Erkrankung, sondern fasst ein breites Spektrum unterschiedlicher Entitäten zusammen. Viele bezeichnen damit die «Rheumatoide Arthritis», aber gerade diese Gleichsetzung ist unzutreffend, da sie praktisch nie mit einer intraokularen Entzündung einhergeht.

\section{Ergebnisse der Studie}

Elvis Hysa und Mitarbeiter stellen in einer lesenswerten Übersichtsarbeit Grundlagen zur Immunologie intraokularer Entzündungen dar. Dabei fokussieren die Autoren auf drei der häufigsten rheumatologischen Erkrankungen und ihren Bezug zur Uveitis. Alle Systemerkrankungen verlaufen überwiegend chronisch rezidivierend und können irreversible Visusminderung hinterlassen. Interessanterweise sind diese Krankheitsbilder oft nicht unmittelbar (allein) mit Schädigung des Bewegungsapparates assoziiert. Es handelt sich im Einzelnen um den Morbus Behçet, die Spondylarthropathie und die Sarkoidose. Lesenswert ist die Arbeit vor allem im Hinblick auf die doch unterschiedlichen zugrundeliegenden Immunmechanismen. Unterschiede, die sich nicht nur im klinischen Erscheinungsbild der Erkrankungen, sondern auch in ihrer Behandlung ausdrücken.

Die ersten differenzierten Hinweise zur Immunpathologie der intraokularen Entzündung werden auf die tierexperimentelle Autoimmun-Uveitis (EAU) zurückgeführt. Sie belegen eine entscheidende Rolle der spezifischen Immunantwort. Erhöhte Konzentrationen von Interleukin (IL)-2 und Interferon gamma (IFN- $\gamma$ ) wurden in den drainierenden Lymphknoten nachgewiesen, was auf eine Hauptreaktion von T-Helfer (Th) 1 hindeutet. Darüber hinaus stellen IL17-produzierende CD4+-T-Zellen, definiert als Th17-Lymphozyten, Immunzellen dar, die mit der Induktion der EAU assoziiert sind. Umgekehrt waren Th-2-Typ-Antworten mit der Auflösungsphase von EAU assoziiert. Überhaupt wird der «Down Regulation» und Homöostase des Immunsystems nach aktiver Entzündung inzwischen verstärkt Aufmerksamkeit gezollt. In diesem Zusammenhang wurde gezeigt, dass vor allem (CD)4+CD25+ regulatorische T (Treg)-Zellen eine Schlüsselrolle bei der Regression der EAU und eine entscheidende immunmodulatorische Rolle spielen.

\section{Morbus Behçet - Uveitis}

Wie finden sich diese Veränderungen bei den erwähnten Krankheitsbildern wieder? Ähnlich zur EAU besteht beim Morbus Behçet eine vorherrschende okuläre Immuninfiltration aus T-Zellen. Es wird vermutet, dass die Uveitis bei diesen Patienten durch eine Reaktion auf ein bisher unbekanntes Antigen bei Patienten mit genetischer oder epigenetischer Prädisposition hervorgerufen wird. Immer wieder wurden als Auslöser mikrobielle Antigene (u.a. Streptokokken, Helicobacter pylori) oder auch Heat-Shock-Proteine, z.B. HeatShock-Protein 65 (HSP65), angeführt.
Neben einer Th1-Polarisation der Immunantwort liegen zunehmend Hinweise vor, dass auch Th17- und Th22-Lymphozyten eine wichtige Rolle spielen. Im peripheren Blut und auch im Kammerwasser konnten bei aktiver Uveitis erhöhte IL-17-Konzentrationen nachgewiesen werden. Interessant und bisher nur bei MorbusBehçet-Patienten wurden intraokular Th22-Typ-T-Zell-Klone in hohen Mengen im Kammerwasser aktiver Patienten nachgewiesen, und die IL-22-Spiegel korrelierten mit der Schwere der retinalen Vaskulitis.

Da auch eine Reihe von Hinweisen für eine sehr aktive Rolle des angeborenen Immunsystems vorliegt, bleiben viele Fragen der Pathophysiologie offen. Fragen, die im Hinblick auf eine gezieltere Behandlung der Vaskulitis wichtig sind.

\section{Spondyloarthropathie - Uveitis}

Die intraokulare Entzündung ist die häufigste extraartikuläre Manifestation der seronegativen Spondyloarthropathie (SpA). Es handelt sich hier um eine ganze Familie von entzündlich-rheumatischen Erkrankungen, die gemeinsame klinische Merkmale aufweisen. Neben einer axialen und peripheren entzündlichen Arthritis besteht eine starke Assoziation zum HLA-B27-Epitop. Besonderes «Augenmerk» gilt es bei HLA-B27+ Uveitis weiteren Erkrankungen aus diesem Formenkreis zu schenken. Zu nennen sind insbesondere die Psoriasis-Arthritis, reaktive Arthritis aber auch entzündliche Darmerkrankungen.

Der Darm-Augen-Achse und dem spezifischen Mikrobiom wird aktuell große Aufmerksamkeit geschenkt. HLA-B27 transgene Mäuse entwickeln keine Uveitis oder Arthritis, wenn sie in einer keimfreien Umgebung gehalten werden.

Bisherige Erkenntnisse und Vermutungen über die Zusammenhänge werden in dem interessanten Artikel vorgestellt und reichen von molekularer Mimikry bis zur gestörten Induktion von Treg-Zellen durch Darmbakterien. Unmittelbare Konsequenzen lassen sich daraus bisher nicht ableiten.

Als wirksam in der Behandlung dieser Systemerkrankungen mit assoziierter Uveitis haben sich vor allem TNF-Antagonisten bewährt. Auch die IL-23/IL-17-Achse scheint für Gelenk- und Hautbehandlung ein attraktives therapeutisches Ziel zu sein. Entsprechende Studien mit Behandlungsindikation Uveitis sind eingeleitet, u.a. eine Phase-II-Studie mit Ustekinumab, einem IL-12/IL23-Blocker.

\section{Sarkoidose - Uveitis}

Zu den Erkrankungen, die einer interdisziplinären Betreuung bedürfen, zählt auch die Sarkoidose. Sie verläuft als chronisch granulomatöse Erkrankung, die typischerweise Lunge, intrathorakale Lymphknoten, Augen und Haut betrifft. Wie bei den beiden zuerst genannten Erkrankungen besteht u.a. auch hier eine Prädisposition mit Assoziation zu bestimmten HLA-Genen (HLA-DR3) und NichtHLA-Polymorphismen.

Das verursachende Antigen ist bisher unklar. Es wird bisher vermutet, dass eine kreuzreagierende Immunantwort die Freisetzung von 
TNF-a, IL-12, IL-15, IL-18, MIP-1, und Granulozyten-Makrophagen-KoIonie-stimulierenden Faktor (GMCSF) verursacht. Die Immunpolarisation von Th1 wurde sowohl durch diagnostische Eingriffe wie der bronchoalveolären Lavage (BAL) als auch indirekt bei therapeutischen Maßnahmen nachgewiesen. So führt die Behandlung mit IFN-a durch den Th-1-förderndernden Effekt zu neuen oder rezidivierenden sarkoidalen Läsionen. Unter den adaptiven Immuneffektoren bei der Granulom-Bildung spielen auch Th17-Zellen eine Schlüsselrolle. Sowohl in der klassischen Variante von IL-17-sezernierenden Zellen als auch durch den Th17.1-Phänotyp, bei denen es sich um IFN- $\gamma$-produzierende Lymphozyten handelt. Interessanterweise wurde bei Patienten mit okulärer Sarkoidose eine erhöhte Expression des IL-17-Rezeptors (IL17R) auf CD8+ T-Zellen im peripheren Blut gefunden.

Die charakteristischen Granulome der Sarkoidose bestehen aus Makrophagen, Epithelzellen, Riesenzellen und T-Zellen. Sie können unter anderem auch in Uvea-Gewebe wie z.B. der Aderhaut des Auges persistieren oder zur Fibrose führen. Unter den adaptiven Immuneffektoren spielen bei der Granulom Bildung auch Th17-Zellen eine Rolle. Interessanterweise wurde bei Patienten mit okulärer Sarkoidose eine erhöhte Expression des IL-17-Rezeptors (IL17R) auf CD8+ T-Zellen im peripheren Blut gefunden.
Die anteriore Uveitis ist die häufigste okuläre Manifestation bei Sarkoidose. Funktionell bedeutsam ist jedoch vor allem die Beteiligung am hinteren Augensegment. Hier werden nicht nur neurologische Beteiligungen häufiger gesehen. Bei retino-vaskulärer Erkrankung erhöht sich auch das Risiko für schwere kardiovaskuläre Komplikationen.

\section{Fazit für die Praxis}

Zusammengefasst illustriert die vorliegende Übersichtsarbeit die Bedeutung immunpathologischer Veränderungen sowohl für die intraokulare Entzündung als auch deren Rolle für die multiplen Organbeteiligungen bei rheumatologischen Erkrankungen. Dies geht weit über eine akademische Wissensvermittlung hinaus, da dadurch das therapeutische Ansprechen heutiger Behandlungsoptionen klar wird. Zudem kann davon ausgegangen werden, das weitere zunehmend spezifischere Therapiemöglichkeiten zu erwarten sind.

Korrespondenz an:

Uwe Pleyer, uwe.pleyer@charite.de 